\title{
Kontinuierliche Hebammenbetreuung für die Zeit von Schwangerschaft, Geburt und Wochenbett durch dieselbe Hebamme
}

Forster D, McLachlan H, Davey M-A, et al. Continuity of care by a primary midwife (caseload midwifery) increases women `s satisfaction with antenatal, intrapartum and postpartum care: results from the COSMOS randomized controlled trial. BMC Pregnancy and Childbirth 2016; 16:28. doi 10.1186/ s12884016-0798-y

\section{Ziel der Studie}

Ziel der Studie war es, festzustellen, ob die kontinuierliche Betreuung von Frauen in der Schwangerschaft, unter der Geburt und im Wochenbett durch ein und dieselbe Hebamme (caseload midwifery), verglichen mit einer Standardbetreuung, die Zufriedenheit der Frauen mit der gesamten Betreuung erhöht.

\section{Methodik}

Design: zweiarmige randomisierte kontrollierte Studie

Ein- und Ausschlusskriterien: Probandinnen waren schwangere Frauen, die sich im Royal Women's Hospital in Melbourne zur Geburt angemeldet hatten. Einschlusskriterien waren das
Beherrschen der englischen Sprache, eine bestehende Einlingsschwangerschaft, eine Schwangerschaftsdauer unter 24 Wochen zum Zeitpunkt der Aufnahme in die Studie und ein geringes geburtshilfliches Risiko. Frauen, bei denen eine Sectio caesarea geplant war, wurden aus der Studie ausgeschlossen.

Interventionen: Es erfolgte eine Randomisierung der Frauen in zwei Gruppen. In beiden Gruppen waren NiedrigrisikoSchwangere, die in Alter, Parität und sozialem Status vergleichbar waren. Beide Gruppen wurden bis auf die Intervention gleichbehandelt. Die Intervention selbst bestand aus einer kontinuierlichen Hebammenbetreuung während der Schwangerschaft, unter der Geburt und während des Wochenbetts durch immer dieselbe Hebamme (caseload midwifery) oder maximal eine zweite sogenannte „back up“ Hebamme. Die Frauen der Kontrollgruppe erhielten für die Zeit der Schwangerschaft, der Geburt und des Wochenbetts die in Australien übliche Standardbetreuung, die arzt- oder hebammengeleitet sein konnte, jedoch keine kontinuierliche Betreuung durch ein und dieselbe Person gewährleistete.

Messungen: Die Datenerhebung erfolgte zwei Monate nach der Geburt in Form eines Fragebogens, der per Post zugesandt wurde. Im Fragebogen wurde die Zufriedenheit der Frauen mit der Betreuung, die sie in der Schwangerschaft, während der Geburt und im Wochenbett erhalten hatten, mithilfe einer Likert Skala 
abgefragt. Zusätzlich mussten die Frauen weitere Fragen zur Anzahl der Treffen mit der Hebamme während der einzelnen Betreuungsabschnitte beantworten.

\section{Ergebnisse}

Insgesamt wurden 2.314 Frauen in die Studie eingeschlossen. Die Rücklaufrate der Fragebögen betrug bei der Caseload-Gruppe (Interventionsgruppe) $88 \%$ und bei der Standardgruppe (Kontrollgruppe) $74 \%$. Verglichen mit der Standardgruppe bestand bei der CaseloadGruppe, die die kontinuierliche Betreuung durch ein und dieselbe Hebamme erhielt, eine signifikant höhere Zufriedenheit mit der Betreuung in allen drei Betreuungsabschnitten.

\section{Schlussfolgerung der Autorinnen / Autoren}

Die Autorinnen schlussfolgern, dass Frauen mit geringem geburtshilflichen Risiko zufriedener mit der Betreuung während Schwangerschaft, Geburt und Wochenbett sind, wenn sie während dieser Zeit kontinuierlich von ein und derselben Hebamme betreut werden.

\section{Kommentar}

Die vorliegende Studie ist eine Untersuchung, die im Rahmen der bekannten australischen COSMOS-Studie von 2012 stattgefunden hat, welche der Hypothese nachging, dass eine kontinuierliche
Hebammenbetreuung die Sectiorate senken kann. Die obige Studie bezog sich auf die Annahme, dass caseload midwifery zusätzlich die Zufriedenheit der Frauen mit der Betreuung erhöht.

In dieser Studie wurden die Hebammen und die Frauen aufgrund der Interventionsart nicht verblindet. Dadurch bestand die Möglichkeit, dass die Hebammen der CaseloadGruppe engagierter bei der Betreuung der Frauen waren, sodass die höhere Zufriedenheit dieser Gruppe nicht an der Kontinuität an sich lag, sondern an den stärker motivierten Hebammen. Zudem waren Erstgebärende in der Studie überrepräsentiert (ca. $70 \%$ in jedem Arm), sodass die Aussagekraft für Mehrgebärende geringer ist. Probandinnen der Studie waren zu einem großen Teil Frauen mit Partner aus der (höheren) Mittelschicht. Somit waren Frauen aus niedrigen sozialen Schichten unterrepräsentiert.

Auch die Definition von „Zufriedenheit“ ist individuell sehr unterschiedlich, daher kann es problematisch sein, Zufriedenheit in Form von Skalen in Fragebögen zuverlässig zu messen.

Dennoch hat die Studie eine hohe Aussagekraft für das Setting, in dem sie durchgeführt wurde. Die Müttergesundheitsdienste in Australien drängten damals auf eine Einführung von caseload midwifery und erhielten mit dieser Studie eine Argumentationshilfe. Auch wenn aufgrund unterschiedlicher Strukturen im Gesundheitssystem eine Übertragbarkeit von caseload midwifery auf den deutschsprachigen Raum nicht vollumfänglich möglich ist, können die Ergebnisse dieser Studie auch bei uns als Argumentationshilfe genutzt werden, um Forderungen nach einem Ausbau der Hebammenbetreuung zu untermauern.

Fazit: Die Studie belegt die hohe Bedeutung einer umfassenden Hebammenbetreuung.

\section{Autorinnen / Autoren}

Alexa Schrader ist Hebamme und Berufspädagogin für Gesundheitsberufe B.A. Derzeit studiert sie im Masterstudiengang „Salutophysiologie für Hebammen “ an der FH Salzburg.

\section{Korrespondenzadresse}

E-Mail: alexaschrader@gmx.de

Bibliografie

DOI https://doi.org/10.1055/a-1205-6429

Die Hebamme 2020; 33: 13-14

(c) Georg Thieme Verlag KG Stuttgart .

New York

ISSN 0932-8122 